\title{
A NEW FAMILY OF ENNEPER TYPE MINIMAL SURFACES
}

\author{
YI FANG
}

(Communicated by Jonathan M. Rosenberg)

\begin{abstract}
An Enneper type surface is a complete immersed minimal surface in $\mathbf{R}^{3}$ with only one end and finite total curvature. In this paper we construct a family of Enneper type surfaces of genus 1 , total curvature $-8(2 n+1) \pi, n=$ $0,1,2, \cdots$. We use the Weierstrass $\wp$ elliptic function as a tool and also prove some results about $\wp$ on a square torus.
\end{abstract}

\section{INTRODUCTION}

An Enneper type minimal surface is a complete immersed minimal surface with finite total Gauss curvature and only one end; (i.e., conformally it is a closed genus $k$ Riemannian surface with one puncture). The simplest example is Enneper's surface. It has genus 0 and total curvature $-4 \pi$. There is also a family of genus 0 examples with total curvature $-4 n \pi, n=1,2,3, \cdots$. In [2] Chen and Gackstätter constructed genus 1 and genus 2 examples with total curvature $-8 \pi$ and $-12 \pi$. In [6] Wohlgemuth constructed a family of genus 1 examples with total curvature $-4 \pi(2 n+1)$ for $n \geq 1$. In this paper we will construct a family of genus 1 examples with total curvature $-8 \pi(2 n+1)$ for $n \geq 0$. Our main tools are Weierstrass representations for minimal surfaces and the Weierstrass elliptic function $\wp$ associated to a lattice $L=[1, \tau]$ in the complex plane $\mathbf{C}$. The by-products of this study are some properties of the Weierstrass $\wp$ function. Having not seen these properties in publication, we list them as a theorem in this paper.

\section{WEIERSTRASS REPRESENTATION}

A very important tool used in the construction of minimal surfaces is the Weierstrass representation formula. Here we state one version of it; for details see [4] and [5].

Received by the editors January 23, 1989 and, in revised form, August 22, 1989.

1980 Mathematics Subject Classification (1985 Revision). Primary 53A10; Secondary 30C15.

The research described in this paper was supported by research grant DE-FG02-86ER250125 of the Applied Mathematical Science subprogram of the Office of Energy Research, U. S. Department of Energy, and National Science Foundation grant DMS-8802858. 
Proposition 1. Let $\bar{M}$ be a compact Riemann surface and $M=\bar{M}-\left\{p_{1}, \cdots, p_{n}\right\}$. Suppose $\bar{g}: \bar{M} \rightarrow \mathbf{C} \cup\{\infty\}$ is a meromorphic function and $\eta$ is a meromorphic 1-form such that whenever $g=\bar{g} \mid M$ has a pole of order $k$, then $\eta$ has a zero of order $2 k$ and $\eta$ has no other zeros on $M$. Let

$$
\omega_{1}=\frac{1}{2}\left(1-g^{2}\right) \eta, \quad \omega_{2}=\frac{i}{2}\left(1+g^{2}\right) \eta, \quad \omega_{3}=g \eta .
$$

If for any closed curve $\gamma$ in $M$,

$$
\operatorname{Re} \int_{\gamma} \omega_{i}=0, \text { for } i=1,2,3,
$$

then the surface $S$, defined by $X: M \rightarrow \mathbf{R}^{3}$, is a regular minimal surface, where

$$
X(z)=\operatorname{Re}\left(\int_{z_{0}}^{z} \omega_{1}, \int_{z_{0}}^{z} \omega_{2}, \int_{z_{0}}^{x} \omega_{3}\right) .
$$

Here, $z_{0}$ is a fixed point of $M$. Moreover, if at any deleted point $p_{i}$, one of $\omega_{1}, \omega_{2}, \omega_{3}$ has a pole of order at least 2 , then $S$ is also complete. The total curvature of $S$ is

$$
C(S)=-4 \pi m,
$$

where $m$ is the degree of $\bar{g}$.

Proof. See, for example, [4, pp. 112-113] and [5, p. 82], Theorem 9.2.

\section{WEIERSTRASS $\wp$ ELLIPTIC FUNCTION}

Let $L=\left[\omega_{1}, \omega_{2}\right]$ be a lattice $\mathbf{C}$. Associated to each $L$ there is a doubly periodic meromorphic function, the Weierstrass $\wp$ function. It is

$$
\wp(z)=\frac{1}{z^{2}}+\sum_{\omega \neq 0}\left(\frac{1}{(z-\omega)^{2}}-\frac{1}{\omega^{2}}\right),
$$

where $\omega=m \omega_{1}+n \omega_{2}$ for all $(m, n) \in \mathbf{Z} \times \mathbf{Z}$ and $(m, n) \neq(0,0)$. It is easy to see $\wp$ is an even function. Moreover, we have

\section{Lemma 1.}

1. $\wp^{(2 k)}$ is an even function, $\wp^{(2 k+1)}$ is an odd function, where $\wp^{(n)}$ denotes the $\mathrm{n}$ th derivative of $\wp, n \geq 0$.

2. $\wp^{(2 k)}=P_{k}(\wp)$ and $\wp^{(2 k+1)}=Q_{k}(\wp) \wp^{\prime}$. Where $P_{k}$ and $Q_{k}$ are polynomials.

3. If $L=[1, \tau]$ and $\tau \in i \mathbf{R}$ then $\wp(\bar{z}+i)=\overline{\wp(z)}$ and $\wp(-\bar{z}+1)=\overline{\wp(z)}$. Also,

$$
\int_{0}^{1}\left[\wp^{(n)}\right]^{2}\left(\frac{\tau}{2}+t\right) d t>0
$$

for any $n \geq 0$. Furthermore,

$$
\int_{0}^{1}\left[\wp^{(n)}\right]^{2}\left(\frac{\tau}{2}+t\right) \wp^{m}\left(\frac{\tau}{2}+t\right) d t
$$


and

$$
\int_{0}^{1} \wp^{m}\left(\frac{\tau}{2}+t\right) d t
$$

are nonzero real numbers, for any $n, m \in \mathbf{Z}$ and $n \geq 0$.

4. If $L=[1, i], \omega=\frac{1+i}{2}$, then $\wp(\omega+i \bar{z})=\overline{\wp(\omega+z)}, \wp(\omega-i \bar{z})=$ $-\overline{\wp(\omega+z)}$, and $\wp(w+\bar{z})=\overline{\wp(\omega+z)}$. $\wp$ has a double pole at 0, a double zero at $\omega$, and no other zeros or poles. Furthermore,

$$
\begin{gathered}
\wp^{(n)}\left(\frac{i}{2}+t\right)=-i^{n} \wp^{(n)}\left(\frac{1}{2}+i t\right) . \\
{\left[\wp^{(n)}\right]^{2}\left(\frac{i}{2}+t\right)=(-1)^{n}\left[\wp^{(n)}\right]^{2}\left(\frac{1}{2}+i t\right) \quad \text { for } 0 \leq t \leq 1 .}
\end{gathered}
$$

Proof. See [1, pp. 658 and 631], and [6, pp. 17-18].

\section{MiNIMAL SURFACES OF ENNEPER TYPE WITH GENUS 1}

Theorem 1. There is a family of Enneper type surfaces of genus 1 and total curvature $-8 \pi(2 n+1), n=0,1,2, \cdots$.

Proof. We consider the square lattice $L=[1, i]$ in the complex plane C. By Lemma 1 , we have $\wp^{(2 k+1)}(z)=Q_{k}(\wp(z)) \wp^{\prime}(z)$, where $Q_{k}(x)$ is a polynomial. Let $Q_{k}(x)=x^{n} R_{k}(x)$ such that $R_{k}(x)=a_{0}+a_{1} x+\cdots+a_{l} x^{l}$ and $a_{0} \neq 0$. Then the degree of $Q_{k}$ is $n+l=d$. We will denote $n=n(k)$ to emphasize that $n$ depends on $k$. Since $\wp^{(2 k+1)}$ has only one pole at 0 of order $2 k+3$, and $Q_{k}(\wp) \wp^{\prime}$ has only one pole at 0 of order $2 d+3$; hence $d=k$ and $n(k) \leq k$. Let $M=T-\{0\}=\mathrm{C} / L-\{0\}$. We choose

$$
g(z)=a \frac{\wp^{(2 k+1)}(z)}{\wp^{m}(z)}
$$

where $a \in \mathbf{C}$ is an nonzero constant to be determined and $0 \leq n(k)<m \leq$ $k+1, k \geq 0$. Then $g$ has only poles at 0 of order $2(k-m)+3$ and at $\frac{1+i}{2}$ of order $2(m-n(k))-1$. Hence we know that the degree of $g$ is $2(k-m)+$ $3+2(m-n(k))-1=2(k-n(k))+2$. We choose

$$
\eta=\wp^{2(m-n(k))-1}(z) d z \text {. }
$$

Then $\eta$ is never zero on $M$ except at $\omega=\frac{1+i}{2}$, where $g$ has a pole of order $l=2(m-n(k))-1$, and $\eta$ has an zero of order $4(m-n(k))-2=2 l$. Let

$$
\Psi=\frac{\left[\wp^{(2 k+1)}\right]^{2}(z)}{\wp^{2 n(k)+1}} d z=\frac{1}{a^{2}} g^{2}(z) \eta .
$$

Since $\wp^{2(m-n(k))-1}(z)$ and $\left[\wp^{(2 k+1)}\right]^{2}(z) / \wp^{2 n(k)+1}(z)$ are both even functions, they have no residues at 0 . Now in the Weierstrass representation formula with 
these $g$ and $\eta$, we have

$$
\begin{aligned}
\omega_{1} & =\frac{1}{2}\left(\eta-a^{2} \Psi\right), \\
\omega_{2} & =\frac{i}{2}\left(\eta+a^{2} \Psi\right), \\
\omega_{3} & =g \eta=a \wp^{(2 k+1)}(z) \wp^{m-2 n(k)-1}(z) d z \\
& =a \wp^{(2 k+1)}(z) \wp^{m-2 n(k)-1}(z) d z=a \wp^{m-n(k)-1}(z) R_{k}(\wp(z)) \wp^{\prime}(z) d z .
\end{aligned}
$$

Notice that $\omega_{3}$ has a pole at 0 of order greater or equal to 3. By Proposition 1, this Weierstrass representation will generate an Enneper type minimal surface if equation (1) of Proposition 1 is satisfied. Since $\omega_{3}$ is exact, we do not need to worry about its periods. Now let $\gamma_{1}(t)=i / 2+t, \gamma_{2}(t)=1 / 2+i t, 0 \leq t \leq 1$. Then $\gamma_{1}$ and $\gamma_{2}$ are generators of the fundamental group of $T=\mathrm{C} / L$. Hence it is enough to prove that we can choose an $a$ such that

$$
\operatorname{Re} \int_{\gamma i} \omega_{j}=0,
$$

for $i, j=1,2$. Notice that by Lemma 1 we have

$$
\begin{gathered}
{\left[\wp^{(2 k+1)}\right]^{2}\left(\frac{i}{2}+t\right)=-\left[\wp^{(2 k+1)}\right]^{2}\left(\frac{1}{2}+i t\right),} \\
\wp^{l}\left(\frac{i}{2}+t\right)=(-1)^{l} \wp^{l}\left(\frac{1}{2}+i t\right) .
\end{gathered}
$$

Hence

$$
\begin{aligned}
\int_{\gamma 2} \eta & =\int_{0}^{1} \wp^{2(m-n(k))-1}\left(\frac{1}{2}+i t\right) i d t \\
& =-i \int_{0}^{1} \wp^{2(m-n(k))-1}\left(\frac{i}{2}+t\right) d t=-i \int_{\gamma 1} \eta
\end{aligned}
$$

that is,

$$
\int_{\gamma 2} \eta=-i \int_{\gamma 1} \eta
$$

Also

$$
\begin{aligned}
\int_{\gamma^{2}} \Psi & =\int_{0}^{1} \frac{\left[\wp^{(2 k+1)}\right]^{2}\left(\frac{1}{2}+i t\right)}{\wp^{2 n(k)+1}\left(\frac{1}{2}+i t\right)} i d t \\
& =i \int_{0}^{1} \frac{\left[\wp^{(2 k+1)}\right]^{2}\left(\frac{i}{2}+t\right)}{\wp^{2 n(k)+1}\left(\frac{i}{2}+t\right)} d t=i \int_{\gamma 1} \Psi
\end{aligned}
$$

that is,

$$
\int_{\gamma 2} \Psi=i \int_{\gamma 1} \Psi
$$


By Lemma 1, we know that $\int_{\gamma_{1}} \eta$ and $\int_{\gamma_{1}} \Psi$ are nonzero real numbers, so let $a^{2}=\int_{\gamma 1} \eta / \int_{\gamma 1} \Psi ;$ then $a^{2} \in \mathbf{R}$ and $a^{2} \neq 0$. We have

$$
\begin{aligned}
& 2 \int_{\gamma 1} \omega_{1}=\int_{\gamma 1} \eta-a^{2} \int_{\gamma 1} \Psi=0, \\
& 2 \int_{\gamma 2} \omega_{1}=-i \int_{\gamma 1} \eta-i a^{2} \int_{\gamma 1} \Psi \in i \mathbf{R}, \\
& 2 \int_{\gamma 1} \omega_{2}=i \int_{\gamma 1} \eta+i a^{2} \int_{\gamma 1} \Psi \in i \mathbf{R}, \\
& 2 \int_{\gamma 2} \omega_{2}=i \int_{\gamma 2} \eta+i a^{2} \int_{\gamma 2} \Psi=\int_{\gamma 1} \eta-a^{2} \int_{\gamma 1} \Psi=0 .
\end{aligned}
$$

Hence $\operatorname{Re} \int_{\gamma i} \omega_{j}=0$. By Proposition 1 we get a complete minimal surface with genus 1 and one end. Since the degree of $g$ is $2(k-n(k))+2$, the total curvature is $C(S)=-4 \pi(2(k-n(k))+2)$. Let $d=k-n(k)+1 \geq 1$. The only thing that remains to be proved is that $d$ can be any odd positive integer. The next proposition will complete the proof of this theorem.

Proposition 2. The $n(k)$ defined in Theorem 2 satisfies

$$
n(k)= \begin{cases}0 & \text { if } k \text { is even } \\ 1 & \text { if } k \text { is odd }\end{cases}
$$

Proof. First we look at the formula in Lemma 1 stating that

$$
\wp^{(2 k)}=P_{k}(\wp), \quad \wp^{(2 k+1)}=Q_{k}(\wp) \wp^{\prime} .
$$

$P_{k}$ and $Q_{k}$ are polynomials. Let $Q_{k}(x)=a_{0}^{k}+a_{1}^{k} x+\cdots+a_{k}^{k} x^{k}$. We claim that

1. $a_{j}^{k}=0$ if $k \neq j \bmod 2$, and $a_{j}^{k} \in \mathbf{R}$,

2. $a_{0}^{2 k} \neq 0, a_{1}^{2 k+1} \neq 0$.

Clearly claim 1 and claim 2 imply the proposition.

Now $\wp^{\prime 2}=4 \wp^{3}-g_{2} \wp-g_{3}$, where $g_{2}$ and $g_{3}$ depend on the lattice $L=[1, \tau]$. When $\tau=i$, we have $g_{3}=0$, and $g_{2} \in \mathbf{R}, g_{2} \neq 0$ (see [3], Corollary 3, p. 40). We prove claim 1 by induction. Since $\wp^{\prime}=1 \cdot \wp^{\prime}$, so $Q_{0}=1$, and $\wp^{\prime \prime}=6 \wp^{2}-g_{2} / 2, \wp^{\prime \prime \prime}=12 \wp \wp^{\prime}$, so that $Q_{1}(x)=12 x$. Thus claim 1 is true for $k=0$ and $k=1$. Suppose claim 1 is true for $k=2 n$ and $2 n+1, n \geq 0$.

Then

$$
\wp^{(4 n+1)}=\wp^{\prime} \sum_{j=0}^{n} a_{2 j}^{2 n} \wp^{2 j}, \wp^{(4 n+3)}=\wp^{\prime} \sum_{j=0}^{n} a_{2 j+1}^{2 n+1} \wp^{2 j+1} .
$$


We have that

$$
\begin{aligned}
\wp^{(4 n+4)} & =\wp^{\prime 2} \sum_{j=0}^{n}(2 j+1) a_{2 j+1}^{2 n+1} \wp^{2 j}+\wp^{\prime \prime} \sum_{j=0}^{n} a_{2 j+1}^{2 n+1} \wp^{2 j+1} \\
& =\left(4 \wp^{3}-g_{2} \wp\right) \sum_{j=0}^{n}(2 j+1) a_{2 j+1}^{2 n+1} \wp^{2 j}+\left(6 \wp^{2}-g_{2} / 2\right) \sum_{j=0}^{n} a_{2 j+1}^{2 n+1} \wp^{2 j+1} \\
& =\sum_{j=0}^{n}(4(2 j+1)+6) a_{2 j+1}^{2 n+1} \wp^{2 j+3}-\frac{g_{2}}{2} \sum_{j=0}^{n}(2(2 j+1)+1) a_{2 j+1}^{2 n+1} \wp^{2 j+1} .
\end{aligned}
$$

Hence,

$$
\begin{aligned}
\wp^{(4 n+5)} & =\wp^{\prime}\left\{\sum_{j=0}^{n}(2 j+3)(8 j+10) a_{2 j+1}^{2 n+1} \wp^{2 j+2}\right. \\
& \left.-\frac{g_{2}}{2} \sum_{j=0}^{n}(2 j+1)(4 j+3) a_{2 j+1}^{2 n+1} \wp^{2 j}\right\} \\
& \wp^{\prime}\left\{-\frac{3 g_{2}}{2} a_{1}^{2 n+1}+\sum_{j=1}^{n}(2 j+1)\left[(8 j+2) a_{2 j-1}^{2 n+1}-\frac{g_{2}}{2}(4 j+3) a_{2 j+1}^{2 n+1}\right] \wp^{2 j}\right. \\
& \left.+(2 n+3)(8 n+10) a_{2 n+1}^{2 n+1} \wp^{2 n+2}\right\} \\
& =\wp^{\prime} \sum_{j=0}^{n+1} a_{2 j}^{2 n+2} \wp^{2 j} .
\end{aligned}
$$

Since there are only even terms and all the $a_{j}^{2 n+1}$ and $j$ are real, so $a_{j}^{2 n+2}$ is real and claim 1 is true for $k=2 n+2$. Also we can see by the computation that $a_{0}^{2 n+2}=-\frac{3}{2} g^{2} a_{1}^{2 n+1}$. Similarly,

$$
\begin{aligned}
\wp^{(4 n+6)} & =\wp^{\prime 2} \sum_{j=0}^{n+1} 2 j a_{2 j}^{2 n+2} \wp^{2 j-1}+\wp^{\prime \prime} \sum_{j=0}^{n+1} a_{2 j}^{2 n+2} \wp^{2 j} \\
& =\sum_{j=0}^{n+1}(8 j+6) a_{2 j}^{2 n+2} \wp^{2 j+2}-\frac{g_{2}}{2} \sum_{j=0}^{n+1}(8 j+1) a_{2 j}^{2 n+2} \wp^{2 j}=\sum_{j=0}^{n+1} b_{2 j+2} \wp^{2 j+2},
\end{aligned}
$$

so

$$
\wp^{(4 n+7)}=\wp^{\prime} \sum_{j=0}^{n+1}(2 j+2) b_{2 j+2} \wp^{2 j+1}=\wp^{\prime} \sum_{j=0}^{n+1} a_{2 j+1}^{2 n+3} \wp^{2 j+1} .
$$

Hence $k=2 n+3$ is true for claim 1. This completes the proof of claim 1 .

Notice that $a_{0}^{0}=1, a_{1}^{1}=12$, and $a_{0}^{2 n}=-\frac{3 g_{2}}{2} a_{1}^{2 n-1}$ for $n \geq 1$. So we need only to prove that $a_{0}^{2 n} \neq 0$. Let $\omega=\frac{1+i}{2}$. Since $\wp(\omega)=\wp^{\prime}(\omega)=0$, 
$\wp^{\prime \prime}(\omega) \neq 0$, so $\omega$ is a double zero of $\wp$, a single zero of $\wp^{\prime}$. In (2) of Lemma 1 set $t=1 / 2$ and $n=4 k$. We get $\wp^{(4 k)}(\omega)=-\wp^{(4 k)}(\omega)$, so $\wp^{(4 k)}(\omega)=0$. Also by $\wp^{(2 k+1)}=\wp^{\prime} Q_{k}(\wp)$ we know that $\wp^{(2 k+1)}(\omega)=\wp^{(2 k+1)}\left(\omega+\frac{1}{2}\right)=0$. If $a_{0}^{2 n}=0$, then $\omega$ will be a zero of $\wp^{(4 n+1)}$ of order at least 3 . So if we prove that $\wp^{(4 n+2)}(\omega) \neq 0$, then $\wp^{(4 n+1)}$ will have only a single zero at $\omega$ and thus $a_{0}^{2 n} \neq 0$. We will count the number of zeros for $\wp^{(4 n+2)}$. Let $I$ be the open interval $(0,1 / 2)$. Then on $\omega+I$ (for $\omega+I$ we mean the interval $1 / 2+i / 2+t$, $0<t<1 / 2$, similarly $\omega+i I$ means the interval $1 / 2+i / 2+i t, 0<t<1 / 2)$, $\wp$ and $\wp^{\prime}$ are real, and $\wp(\omega+\bar{z})=\overline{\wp(\omega+z)}, \wp^{\prime}(\omega+\bar{z})=\overline{\wp^{\prime}(\omega+z)}$. By claim 1 , any $\wp^{(k)}, k \geq 0$, has these properties. If $z=x+i y$, then

$$
\left.\frac{\partial \operatorname{Re} \wp^{(k)}(\omega+z)}{\partial y}\right|_{y=0}=0
$$

on $\omega+I$. For a holomorphic function $f$,

$$
f^{\prime}=2(\operatorname{Re} f)_{z}=\frac{\partial \operatorname{Re} f}{\partial x}-i \frac{\partial \operatorname{Re} f}{\partial y},
$$

on $\omega+I$ we have

$$
\wp^{(k+1)}(\omega+x)=\frac{\partial \operatorname{Re} \wp^{(k)}(\omega+x)}{\partial x}-i \frac{\partial \operatorname{Re} \wp(\omega+x)}{\partial y}=\frac{\partial \wp^{(k)}(\omega+x)}{\partial x}
$$

for all $x \in I$. We claim that for any $n \geq 0, \wp^{(4 n+2)}$ has at least $n+1$ different zeros on $\omega+I$. Since $\wp^{\prime}(\omega+1 / 2)=\wp^{\prime}(\omega)=0$, by Rolle's theorem there is at least one $x_{0} \in I$ such that $\wp^{\prime \prime}\left(\omega+x_{0}\right)=0$. Hence for $n=0$ the claim is true. We now apply induction. Suppose for $n=k \geq 0, \wp^{(4 k+2)}$ has at least $k+1$ different zero points on $\omega+I$. Then by Rolle's theorem, $\wp^{(4 k+3)}$ has at least $k$ different zero points on $\omega+I$. Note $\wp^{(4 k+3)}(\omega)=\wp^{(4 k+3)}(\omega+1 / 2)=0$, so on the closure of $\omega+I, \wp^{(4 k+3)}$ has at least $k+2$ different zeros. Again by Rolle's theorem $\wp^{(4 k+4)}$ has at least $k+1$ different zeros on $\omega+I$. Because $\wp^{(4 k+4)}(\omega)=0$, on the closure of $\omega+I, \wp^{(4 k+4)}$ has at least $k+2$ different zeros. Hence $\wp^{(4 k+5)}$ has at least $k+1$ different zeros on $\omega+I$. Again, $\wp^{(4 k+5)}(\omega)=\wp^{(4 k+5)}(\omega+1 / 2)=0$, so that $\wp^{(4 k+6)}$ has at least $k+2$ different zeros on $\omega+I$. Hence we have thus proved this claim. For $x \in I, \wp(\omega+x)=$ $\wp(\omega-x), \wp(\omega+x)=\wp(\omega+i x), \wp(\omega+i x)=\wp(\omega-i x)$. Hence $\wp^{(4 n+2)}$ has at least $4 n+4$ different zeros. But $\operatorname{deg} \wp^{(4 n+2)}=4 n+4$, so $\wp^{(4 n+2)}$ can have only $4 n+4$ zeros. Thus we have found all of the zeros of $\wp^{(4 n+2)}$. Because these zeros are in $\omega+I, \omega-I, \omega+i I, \omega-i I$, we conclude that $\wp^{(4 n+2)}(\omega) \neq 0$. Thus the proof of this proposition is complete.

Remark 1. When setting $k=0$, we get Chen and Gackstäter's genus 1 example.

Remark 2. By the proof of Proposition 2, we get some properties of the Weierstrass $\wp$ function associated to $L=[1, i]$. We list these properties as a separate theorem. 
Theorem 2. The Weierstrass $\wp$ function associated with $[1, i]$ has the following properties:

1. For $n \geq 0$, all the zeros of $\wp^{(n)}$ are in the two lines $\gamma_{1}(t)=i / 2+t$, $\gamma_{2}(t)=1 / 2+i t, 0 \leq t \leq 1$. The zeros are symmetric about $\omega=\frac{1+i}{2}$.

2. For $n \geq 0, \wp^{(4 n)}$ has a double zero at $\omega, \wp^{(4 n+3)}$ has a triple zero at $\omega$. Any other zeros of $\wp^{(n)}$ are simple.

3. $\wp^{(4 n)}(\omega)=\wp^{(4 n+1)}(\omega)=\wp^{(4 n+3)}(\omega)=0$ and $\wp^{(4 n+2)}(\omega) \neq 0$ for $n \geq 0$.

Proof. Just as in the proof of Proposition 1 count the number of zeros on $\omega+I$, using the three symmetries and $\operatorname{deg} \wp^{(k)}=k+2$. Note that because $\wp^{(4 n-1)}(\omega)=\wp^{(4 n)}(\omega)=\wp^{(4 n+1)}(\omega)=0, \wp^{(4 n+2)}(\omega) \neq 0$, we get claim 2 .

\section{ACKNOWLEDGMENTS}

The author would like to thank Professors D. Hoffman and W. Meeks for their invaluable help. It is from them that the author has learned what a minimal surface is. The author's thanks also go to the referee for patient reading and recommendations, and to James Riordan for correcting grammar and spelling mistakes.

\section{REFERENCES}

1. M. Abromovitz and I. Stegun, ed., Handbook of mathematical functions, Advanced Mathematics, Dover Publications, Inc., New York, 1972, chapter 18.

2. C. C. Chen and F. Gackstätter, Elliptische und hyperelliptische Funktionen und vollständige Minimalflächen vom Enneperschen Typ., Math. Ann. 259 (1982), 359-369.

3. S. Lang, Elliptic functions, 2nd ed., Dover Publications, New York, 1987,

4. H. B. Lawson, Jr., Lectures on minimal submanifolds. Publish or Perish Press, Berkeley, 1971.

5. R. Osserman, A Survey of minimal surfaces, 2nd ed., Dover Publications, New York, 1986.

6. M. Wohlgemuth, Abelsche Minimalflächen, Diplomarbeit, Universität Bonn, 1988.

Department of Mathematics and Statistics, University of Massachusetts at AmHerst, AMHERST, MASSACHUSETTS 01003 